\title{
Cytotoxic and Mutagenic Properties of C3'-Epimeric Lesions of 2'-Deoxyribonucleosides in Escherichia coli Cells
}

\author{
Pengcheng Wang ${ }^{\dagger}$, Nicholas J. Amato ${ }^{\ddagger}$, and Yinsheng Wang ${ }^{\star}, \dagger, \neq, \mathrm{iD}$ \\ tEnvironmental Toxicology Graduate Program, University of California, Riverside, California \\ 92521-0403, United States \\ ‡Department of Chemistry, University of California, Riverside, California 92521-0403, United \\ States
}

\section{Abstract}

Reactive oxygen species (ROS), resulting from endogenous metabolism and/or environmental exposure, can induce damage to the 2-deoxyribose moiety in DNA. Specifically, a hydrogen atom from each of the five carbon atoms in 2-deoxyribose can be abstracted by hydroxyl radical, and improper chemical repair of the ensuing radicals formed at the $\mathrm{C}^{\prime}{ }^{\prime}, \mathrm{C}^{\prime}$, and $\mathrm{C}^{\prime}{ }^{\prime}$ positions can lead to the stereochemical inversion at these sites to yield epimeric 2-deoxyribose lesions. Although ROS-induced single-nucleobase lesions have been well studied, the biological consequences of the $\mathrm{C} 3^{\prime}$-epimeric lesions of $2^{\prime}$-deoxynucleosides, i.e., $2^{\prime}$-deoxyxylonucleosides $(\mathrm{dxN})$, have not been comprehensively investigated. Herein, we assessed the impact of $\mathrm{dxN}$ lesions on the efficiency and fidelity of DNA replication in Escherichia coli cells by conducting a competitive replication and adduct bypass assay with single-stranded M13 phage containing a sitespecifically incorporated $d x N$. Our results revealed that, of the four $d x N$ lesions, only $d x G$ constituted a strong impediment to DNA replication, and intriguingly, $\mathrm{dxT}$ and $\mathrm{dxC}$ conferred replication bypass efficiencies higher than those of the unmodified counterparts. In addition, the three SOS-induced DNA polymerases (Pol II, Pol IV, and Pol V) did not play any appreciable role in bypassing these lesions. Among the four dxNs, only dxA directed a moderate frequency of dCMP misincorporation. These results provided important insights into the impact of the $\mathrm{C}^{\prime}$ epimeric lesions on DNA replication in E. coli cells.

\section{Graphical abstract}

*Corresponding Author. yinsheng.wang@ucr.edu. Telephone: (951) 827-2700.

ORCID

Yinsheng Wang: 0000-0001-5565-283X

\section{ASSOCIATED CONTENT}

Supporting Information

The Supporting Information is available free of charge on the ACS Publications website at DOI: 10.1021/acs.biochem.7b00146.

Detailed experimental procedures, MS and NMR results for the characterizations of synthetic compounds, and gel images for monitoring the restriction fragments of PCR products of the progeny genome arising from cellular replication (PDF) 


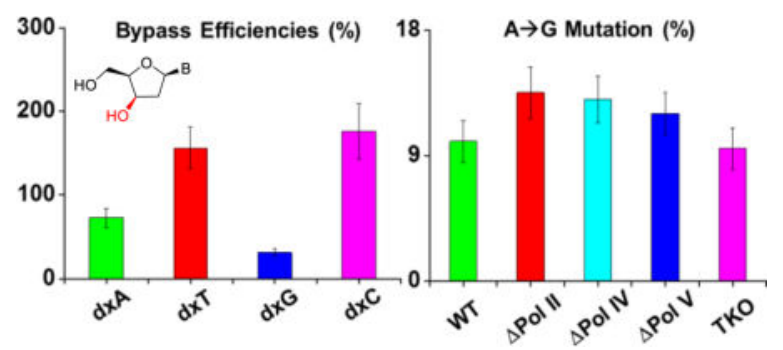

Environmental exposure and aerobic metabolism unavoidably give rise to the generation of reactive oxygen species (ROS), which are required for normal cellular functions, including cell signaling and immune responses. ${ }^{1,2}$ However, when the capacity of antioxidant defense is inadequate to completely scavenge the ROS produced in cells, damage to macromolecules such as lipids, proteins, and DNA may ensue, which may contribute to the pathogenesis of neurological abnormalities, cancer, and other diseases in humans. ${ }^{3-5}$

Oxidatively induced DNA damage has been extensively investigated. Aside from modifications to nucleobases, which are capable of impairing their hydrogen bonding properties, hydroxyl radical can attack the sugar moiety in nucleosides, leading to the formation of 2-deoxyribose radicals. ${ }^{6,7}$ These radicals may induce alkali-labile sites and strand breaks in DNA. ${ }^{6,8}$ Moreover, when hydrogen abstraction occurs at $\mathrm{C1}^{\prime}, \mathrm{C}^{\prime}$, and $\mathrm{C}^{\prime}$ positions, a unique group of lesions, known as epimeric 2-deoxyribose lesions, can be generated via improper chemical repair. ${ }^{7}$ Scheme 1 illustrates the mechanism of formation of the $\mathrm{C} 3^{\prime}$-epimeric lesions of $2^{\prime}$-deoxynucleosides, i.e., $2^{\prime}$-deoxyxylonucleosides (dxNs).

The dxNs were initially shown to form from a photochemically generated $\mathrm{C}^{\prime}$ radical, resulting in a 1:1 mixture of isomers of the $\mathrm{H}$-abstraction products when the photolysis was performed in the presence of a hydrogen atom donor. ${ }^{9}$ In this regard, dxT was found to form in oligodeoxyribonucleotides (ODNs) from the independently generated $\mathrm{C}^{\prime}{ }^{\prime}$ radical under anaerobic conditions in the presence of glutathione (GSH), with the yields being higher in single-stranded DNA and overhang structure than in duplex DNA. ${ }^{10}$ In addition, the formation of the $\mathrm{C}^{\prime}$-epimeric lesion is in direct competition with strand break generation under anaerobic conditions. ${ }^{10,11}$

Not much is known about the occurrence of dxNs in cellular and tissue DNA. Because of the structural similarities of epimeric nucleosides, we found that the $\mathrm{C} 3{ }^{\prime}$-epimeric lesions could not be resolved from their $\mathrm{C}^{\prime}$ counterparts on LC columns with several types of stationaryphase materials (ref 12 and unpublished results). This difficulty, along with the same molecular weight and the same fragmentation patterns in MS/MS for the $\mathrm{C}^{\prime}{ }^{\prime}$ - and $\mathrm{C} 4{ }^{\prime}$ epimeric lesions, renders it a significant analytical challenge to detect the $\mathrm{C} 3^{\prime}$-epimeric lesions without interference from the $\mathrm{C}^{\prime}{ }^{\prime}$ counterparts. Nevertheless, a previous study by Tullius and co-workers ${ }^{13}$ showed that the hydroxyl radical-mediated hydrogen atom abstraction from the 2-deoxyribose occurs in the following order: $5^{\prime} \mathrm{H}>4^{\prime} \mathrm{H}>3^{\prime} \mathrm{H} \approx 2^{\prime}$ $\mathrm{H} \approx 1^{\prime} \mathrm{H}$. Considering that the $\mathrm{C} 1^{\prime}$ epimer of $\mathrm{dG}$ could be detected at appreciable levels in DNA of mammalian tissues, ${ }^{12}$ it is highly likely that $\mathrm{C} 3^{\prime}$ epimers are also present in mammalian tissues. In this vein, we observed the presence of appreciable levels of the C3' 
and/or $\mathrm{C}^{\prime}$ ' epimers of $\mathrm{dG}$ in mammalian tissue DNA, though the relative levels of the $\mathrm{C}^{\prime}$ and $\mathrm{C}^{\prime}$ epimers of $\mathrm{dG}$ could not established. ${ }^{12}$

The chemical modification at the $\mathrm{C}^{\prime}$ position of 2-deoxyribose leads to an alteration of sugar puckering and backbone torsion, which results in perturbations of DNA stability and three-dimensional structure. ${ }^{14-21}$ Indeed, the thermal stability of the duplexes was substantially diminished upon the introduction of a few xylose nucleosides into one strand of DNA ${ }^{14-17}$ or RNA; ${ }^{20,21}$ however, complete modification of one strand abrogated duplex formation. ${ }^{14-17}$ Interestingly, duplexes formed from self-complementary fully modified xylose-DNA (dXNA) exhibit stabilities similar to or even greater than those of the corresponding DNA duplexes. ${ }^{17,19}$ It is also noteworthy that when dXNA pairs with other DNA and/or RNA, they tend to form triplexes with a stability comparable to that of the natural counterparts. ${ }^{21-24}$ Additionally, in self-complementary fully modified dXNA, purine bases $(A / G)$ were found to introduce backbone distortion larger than that of pyrimidine bases (T/C).${ }^{19}$ Furthermore, studies have been conducted to assess the influence of individually incorporated dxNs on the thermal stability of duplex DNA, ${ }^{14-17}$ though the experiments for the four dxNs were not performed with the use of the same DNA sequence context.

Not much is known about the biological end points of $\mathrm{C} 3^{\prime}$-epimeric lesions. It was demonstrated that several DNA polymerases were capable of utilizing $2^{\prime}$ deoxyxylonucleoside triphosphates (dxNTPs) during primer extension, though the elongation was incomplete. ${ }^{19}$ In addition, dxN-containing double-stranded DNA was resistant to nucleases. ${ }^{14,15,25}$ Nevertheless, little is known about how this type of lesion affects the flow of genetic information by compromising DNA replication and transcription. Herein, we set out to examine comprehensively how the fidelity and efficiency of DNA replication are perturbed by dxN lesions and how SOS-induced DNA polymerases contribute to the replicative bypass of these lesions in Escherichia coli cells.

\section{EXPERIMENTAL PROCEDURES}

\section{Materials}

All solvents and reagents, unless otherwise noted, were purchased from Sigma-Aldrich (St. Louis, MO) or Thermo Fisher Scientific (Waltham, MA). Unmodified oligodeoxyribonucleotides (ODNs) were obtained from Integrated DNA Technologies (Coralville, IA), and reagents for solid-phase DNA synthesis were purchased from Glen Research Co. (Sterling, VA). All enzymes were from New England Biolabs (Ipswich, MA), and $\left[\gamma_{-}{ }^{32} \mathrm{P}\right]$ ATP was obtained from PerkinElmer (Piscataway, NJ). The M13mp7(L2) phage, wild-type, and polymerase-deficient AB1157 E. coli strains used in this study were kindly provided by J. M. Essigmann and G. C. Walker. ${ }^{26}$

\section{Preparation of Lesion-Carrying ODNs}

The synthetic routes for $2^{\prime}$-deoxyxylonucleoside $(\mathrm{dxN})$ phosphoramidites were adapted from previously published procedures (Scheme 2) ${ }^{27}$ Detailed synthetic procedures, reaction yields, and NMR spectra are provided in Figures S1-S16. 
The synthesized phosphoramidite building blocks were then employed for the site-specific incorporation of dxN into 12-mer ODNs, 5'-ATGGCGXGCTAT-3' (X = dxN, GXG) and $5^{\prime}$-ATGGCXTCTAT-3' $(X=\mathrm{dxC}, \mathrm{CXT})$, via solid-phase DNA synthesis on a Beckman (Fullerton, CA) Oligo 1000S DNA synthesizer, following the vendor's recommended protocols.

The synthesized 12-mer ODNs were deprotected and cleaved from a solid-phase support with 30\% ammonium hydroxide, purified by high-performance liquid chromatography (HPLC), and characterized by ESI-MS and MS/MS, as described elsewhere. ${ }^{12,28,29}$ HPLC traces are displayed in Figure S17, and the MS and MS/MS data are shown in Figures S18$\mathrm{S} 22$. The purified 12-mer dxN-containing ODNs were then ligated individually with a 10mer ODN (5'-AGTGGAAGAC-3') using T4 DNA ligase, following standard protocols. The desired 22-mer lesion-bearing ODNs were then isolated from the reaction mixture via denaturing polyacrylamide gel electrophoresis (PAGE).

\section{Construction of Single-Stranded Lesion-Bearing and Lesion-Free Competitor M13 Genomes and Their Transfection into E. coli Cells}

The preparation of single-stranded (ss) M13mp7(L2) genomes was performed according to previously reported procedures (Figure S23). ${ }^{30}$ Briefly, the EcoRI-linearized ssM13 genome was annealed with two scaffolds, $5^{\prime}$-CTTCCACTCACTGAATCATGGTCATAGCTTTC-3' and $5^{\prime}$-AAAACGACGGCCAGTGAATTATAGC-3' (25 pmol), together with $5^{\prime}$ phosphorylated 22-mer lesion-carrying ODNs, the control lesion-free ODNs, or the 25-mer competitor ODN (30 pmol each). After ligation, the scaffolds and the unligated vector were removed by using T4 DNA polymerase, and the mixture was purified using a Cycle Pure Kit (Omega) to afford circular lesion-containing and lesion-free M13 genomes. Accurate concentrations of the constructed lesion-containing and lesion-free genomes were determined by normalizing against the competitor genome, as described previously (Figure S24). ${ }^{30}$

Transfection was performed in AB1157 E. coli cells that are proficient or deficient in Pol II, Pol IV, Pol V, or all three polymerases, with or without SOS induction, ${ }^{26}$ following the previously published procedures. ${ }^{30}$ The lesion:competitor or control:competitor genome ratio is 1:1. The progeny genome was isolated with QIAprep Spin M13 kit (Qiagen) and amplified by polymerase chain reaction (PCR).

\section{Assessment of the Bypass Efficiencies and Mutation Frequencies}

A modified competitive replication and adduct bypass (CRAB) assay was used to determine the bypass efficiencies of the $\mathrm{dxN}$ lesions in E. coli cells (Figure 1). ${ }^{31,32}$ The interested region in the purified ssM13 template was amplified by PCR. The resulting PCR products were purified and employed to determine bypass efficiency and mutation frequency via sequential restriction endonuclease digestion and native PAGE analysis (Figure 2). 7,28,29 The detailed procedures are provided in the Supporting Information.

As illustrated in Figure 2, two restriction endonucleases were used, i.e., BbsI and MluCI. When BbsI was added first in the digestion process, 10-mer ODN from the initial lesionsituated strand was radiolabeled (Figure 2a,b), i.e., d(p*GGCGMGCTAT) and 
$\mathrm{d}(\mathrm{p} *$ GGCMTGCTAT) for the GXG and CXT sequences, respectively, with $\mathrm{M}$ being A, T, G, or $\mathrm{C}$ and $\mathrm{p} *$ designating the $5^{\prime}-{ }^{32} \mathrm{P}$-labeled phosphate. For the GXG sequence, the two products with $\mathrm{M}$ being a $\mathrm{C}$ or $\mathrm{T}$ could not be resolved from each other by PAGE analysis (Figure 2c,d). Hence, the order for the additions of the two restriction enzymes was reversed to radiolabel the 10-mer strand released from the opposite strand (Figure 2a). Under this circumstance, the sequences became d(AATTATAGCN), with $\mathrm{N}$ being a $\mathrm{G}$ or $\mathrm{A}$, which could be readily resolved from each other (Figure 2e), as described previously. ${ }^{12,31,32}$ Thus, this sequential restriction endonuclease digestion and native PAGE method allowed for the complete differentiation of four types of base incorporations, thereby facilitating the quantifications of bypass efficiency and mutation frequency. It should be noted that, for the CXT sequence, only the restriction fragment housing the initial lesion-situated strand was used for analysis (Figure 2b,d), as the base $\mathrm{N}$ inserted opposite the lesion site was not part of the 10-mer restriction fragment.

To obtain sufficient products for LC-MS/MS analysis, larger quantities of PCR products were digested by the two aforementioned restriction enzymes. Detailed procedures are provided in the Supporting Information. The digestion enzymes were removed via phenol/ chloroform extraction, and the top aqueous layer was recovered and concentrated in a Speed-vac. The residue was desalted via HPLC and redissolved in water. A portion of the resulting aliquot was injected for LC-MS/MS analysis under previously described conditions, ${ }^{7,28,29}$ and fragmentations of the $[\mathrm{M}-3 \mathrm{H}]^{3-}$ ions of the released 10-mer ODNs were monitored. For the lesion-situated strand, the 10-mer ODNs d(GGCGMGCTAT) (for the GXG sequence) or d(GGCMTGCTAT) (for the CXT sequence), where M indicates A, T, $\mathrm{G}$, or $\mathrm{C}$, were monitored. The corresponding ODNs d(AATTATAGCN) (for the GXG sequence), with $\mathrm{N}$ being $\mathrm{A}, \mathrm{T}, \mathrm{G}$, or $\mathrm{C}$, were monitored for the opposite strand. The detected fragment ions in the tandem mass spectra were manually assigned (Figure 3 and Figures S31-S35)

\section{RESULTS AND DISCUSSION}

We aim to gain a comprehensive understanding of the impact of dxN lesions on DNA replication and the roles of SOS-induced DNA polymerases in bypassing these lesions in $E$. coli cells. Thus, ODNs harboring site-specifically incorporated $\mathrm{dxN}$ lesions were first prepared and characterized by ESI-MS and MS/MS (Figures S18-S22). As no change in molecular mass was introduced by epimerization at the $\mathrm{C} 3^{\prime}$ site, the MS and MS/MS data of these lesion-bearing ODNs are almost identical to those of the corresponding unmodified ODNs. Realizing that flanking sequences are known to modulate the mutagenic properties of DNA lesions, ${ }^{33,34}$ we synthesized another dxC-containing ODN, i.e., the CXT sequence (see Experimental Procedures).

\section{Replication Perturbation Induced by dxN Lesions in Vivo}

We employed a modified version of the CRAB assay ${ }^{31,32}$ to assess the degrees to which the $\mathrm{dxN}$ lesions impede DNA replication and induce mutations in these processes and to investigate how replication past these lesions is modulated by translesion synthesis DNA polymerases (Figure 1). In this assay, the lesion-containing ODNs mentioned above and the 
corresponding lesion-free ODNs were first ligated individually into the single-stranded M13 plasmid. Because transfection efficiencies can vary, premixing the lesion-bearing or the respective lesion-free genome with a nonlesion competitor genome prior to transfection allows the blockage to replication to be accurately quantified from the attenuation in the ratio of output signal for the lesion/competitor genome relative to that for the corresponding control/competitor genome. In this vein, the competitor genome acts as an internal standard, and the bypass efficiency of the corresponding control lesion-free M13 genome is considered $100 \%$. After replication in E. coli cells, the region of the isolated progeny M13 genomes harboring the initial lesion or corresponding control site was amplified by PCR (Figures 1 and 2). The resulting PCR products were digested with two restriction enzymes, i.e., BbsI and MluCI, and the restriction digestion products were subjected to LC-MS/MS and PAGE analyses (Figures 1-3 and Figures S25-S35).

Our PAGE and LC-MS/MS results showed that the dxN lesions did not give rise to insertion or deletion mutation, where only the 10-mer radiolabeled restriction fragment(s) could be detected for the lesion-containing or lesion-free progeny M13 genome (Figures S25-S29). In this vein, the corresponding digestion of PCR products of the progeny of the competitor genome afforded a 13-mer fragment (Figure 3).

The bypass efficiencies of the $\mathrm{dxN}$ lesions can be calculated from the ratio of the 10-mer to the 13-mer product as described in Experimental Procedures, considering the input molar ratios of the lesion and control over competitor genomes (Figure 4a). It turned out that the two purine nucleosides, i.e., dxA and dxG, exhibit blockage effects on DNA replication in $E$. coli cells, with $\mathrm{dxG}$ displaying the lowest bypass efficiency ( $\sim 30 \%)$ (Figure $4 \mathrm{a})$. Intriguingly, the two pyrimidine nucleosides, namely, $\mathrm{dxC}$ and $\mathrm{dxT}$, conferred replication bypass efficiencies higher than those of the corresponding unmodified nucleosides, and this observation holds true for $\mathrm{dxC}$ in another sequence context (Figure 4a). This finding, in conjunction with the previous observation that xylose nucleosides with purine bases introduce larger backbone distortions than those of their pyrimidine counterparts, ${ }^{19}$ indicates that the size of the nucleobase might modulate the replication bypass efficiencies of the xylose nucleosides. The exact reason underlying the elevated bypass for $\mathrm{dxT}$ and $\mathrm{dxC}$ is unknown and warrants further investigation. In this vein, it is of note that the $\mathrm{C}^{\prime}{ }^{\prime}$-epimeric nucleosides constitute an unusual family of DNA lesions in the fact that there is no structural perturbation of the nucleobase portion. Thus, the inversion of the stereochemistry at the C3' position of the two modified pyrimidine nucleosides may facilitate some type(s) of favorable interaction between the modified nucleosides and replicative DNA polymerase, thereby promoting their bypass during DNA replication.

The roles of the SOS-induced DNA polymerases in bypassing the $\mathrm{dxN}$ lesions were also investigated by performing the replication experiments in E. coli strains deficient in these DNA polymerases, Pol II, Pol IV, and Pol V. Because of the relatively high bypass efficiencies of the epimeric lesions (i.e., at least 30\%), we first conducted the experiments in E. coli cells without SOS induction. Interestingly, when the SOS-induced polymerases were depleted, individually or all three together, no substantial differences in bypass efficiencies were observed for any dxN lesions (Figure 4a), suggesting that these polymerases do not play any role in bypassing the $\mathrm{dxN}$ lesions in E. coli cells. 
We also performed the replication experiments with the use of SOS-induced E. coli cells. In this vein, previous studies showed that the copy numbers of Pol II and Pol IV could be increased by approximately 10-fold upon SOS induction, i.e., from approximately 75 and 250 to 1000 and 2500 molecules per cell, respectively, where the copy number of the replicative polymerase (i.e., Pol III) is $\sim 20$ molecules per cell. ${ }^{35,36}$ Different from Pol II and Pol IV, Pol V could not be detected biochemically in non-SOS-induced E. coli strains, and the level of expression of Pol V increases by $~ 100$-fold after SOS induction. ${ }^{36,37}$ Thus, we also assessed how SOS induction modulates the replication bypass efficiencies and mutation frequencies of the dxN lesions in wild-type E. coli cells and the isogenic Pol V-deficient cells. Our results revealed no significant differences in bypass efficiencies between the SOSinduced cells and the corresponding un-induced cells (Figure S30a).

\section{Mutagenic Properties of $\mathrm{dxN}$ Lesions}

The native PAGE analyses also facilitated the quantification of the mutation frequencies of dxN lesions in AB1157 E. coli strains that are proficient or deficient in translesion synthesis (Figure 4b). As illustrated in Figure 3, for the GXG sequence, selective incorporation of a ${ }^{32} \mathrm{P}$-labeled phosphate to the $5^{\prime}$ end of the lesion-situated strand, i.e., $\mathrm{d}\left(\mathrm{p}^{*}\right.$ GGCGMGCTAT), or its complementary strand, i.e., d(p*AATTATAGCN), can be achieved by switching the order of the two restriction digestion steps. This approach allowed products arising from all four types of base incorporations at the lesion site to be resolved from each other by native PAGE analysis (Figure 2c,e). It is also noteworthy that, for CXT sequence, only the 10-mer restriction fragment for the lesion-situated strand is labeled for analysis because the nucleotide incorporated opposite the lesion site is not included in the complementary 10-mer restriction fragment (Figure 2b,d). The identities of restriction digestion products were further confirmed via LC-MS/MS analysis. Specifically, the higherresolution "ultrazoom" scan MS and MS/MS data for the $[\mathrm{M}-3 \mathrm{H}]^{3-}$ ions of $\mathrm{d}($ GGCGMGCTAT)/d(GGCMTGCTAT) and d(AATTATAGCN) were monitored (Figure 2 and Figures S31-S35), where $\mathrm{M}$ and $\mathrm{N}$ represent the incorporated nucleotides at the original damage site and the opposite site in the complementary strand, respectively.

As illustrated by the PAGE results (Figure 2 and Figures S25-S29) and consistent with the LC-MS/MS analyses (Figure 3 and Figures S30-S35), only dxA introduces a moderate frequency of $\mathrm{A} \rightarrow \mathrm{G}$ mutation ( $\sim 10 \%)$, and the other $\mathrm{dxNs}$ are not mutagenic, suggesting that overall this type of lesions is tolerated well by DNA polymerases. Interestingly, the three SOS-induced polymerases did not modulate the $\mathrm{A} \rightarrow \mathrm{G}$ mutation frequencies induced by dxA (Figure 4b). In addition, the mutagenic properties of dxA in wild-type AB1157 cells or the isogenic Pol V-deficient cells were not affected by SOS induction (Figure S30b). These results indicate that the observed $\mathrm{A} \rightarrow \mathrm{G}$ mutation may arise from the intrinsic structural property of dxA. Likewise, high-fidelity replication across the other three $\mathrm{dxNs}$ was not affected by depletion of Pol II, Pol IV, or Pol V, or all three in combination, or by SOS induction in wild-type or Pol V-depleted cells (Figure S30b).

It is worth comparing the results obtained from this study with what we observed previously for the $\mathrm{C}^{\prime}$ epimer of $2^{\prime}$-deoxynucleosides $\left(a\right.$-dN) ${ }^{12}$ In this vein, all of the $a$-dNs except $a$-dA strongly blocked DNA replication, and both $a$-dC and $a$-dG could direct very high 
frequencies of dTMP misincorporation in un-induced wild-type AB1157 cells. Thus, the inversion of the stereochemical configuration at $\mathrm{C}^{\prime}$ and $\mathrm{C}^{\prime}{ }^{\prime}$ of 2-deoxyribose exerts distinct effects on DNA replication, where the former may promote unusual base pairing, e.g., reverse Watson-Crick or reverse wobble base pairing. ${ }^{12,38}$ The study presented here suggests that the inversion of the stereochemical configuration at $\mathrm{C}^{\prime}$ is unlikely to perturb profoundly the base pairing capabilities of the nucleobase, as reflected by the lack of mutagenic effects of the dxNs, except a moderate frequency of $\mathrm{A} \rightarrow \mathrm{G}$ mutation for $\mathrm{dxA}$. The pronounced effects of $d x G$, but not other $d x N s$, on blocking DNA replication suggest that only dxG may elicit substantial structural distortion of the DNA backbone.

Together, our results revealed that the four $\mathrm{C}^{\prime}$-epimeric lesions elicit distinct effects on DNA replication, with only dxA being moderately mutagenic and $\mathrm{dxG}$ moderately impeding replication. We also found that the cytotoxic and mutagenic properties of these four lesions were not modulated by SOS-induced polymerases or by SOS induction in wild-type or Pol V-deficient cells. Our systematic shuttle vector-based study of the $\mathrm{C}^{\prime}{ }^{\prime}$-epimeric lesions of $2^{\prime}$-deoxyribonucleosides provided important new knowledge about the degrees to which the $\mathrm{dxN}$ lesions perturb the efficiency and accuracy of DNA replication in bacterial cells. It will be important to assess how the $\mathrm{dxN}$ lesions compromise DNA replication in mammalian cells and to examine the occurrence of these lesions in mammalian cells and tissues in the future.

\section{Supplementary Material}

Refer to Web version on PubMed Central for supplementary material.

\section{Acknowledgments}

Funding

This work was supported by the National Institutes of Health (R01 ES025121 and P01 AG043376). N.J.A. was supported by a National Research Service Award institutional training grant (T32 ES018827).

\section{ABBREVIATIONS}

$\begin{array}{ll}\text { ROS } & \text { reactive oxygen species } \\ \text { dxN } & 2^{\prime} \text {-deoxyxylonucleoside } \\ \text { ODN } & \text { oligodeoxyribonucleotide } \\ \text { dXNA } & \text { xylose-DNA } \\ \text { dxNTP } & \text { deoxyxylonucleoside triphosphate } \\ \text { HFIP } & \text { hexafluoroisopropanol } \\ \text { TBDMS } & \text { tert-butyldimethylsilyl } \\ \text { PAGE } & \text { polyacrylamide gel electrophoresis } \\ \text { TBAF } & \text { tetra- } n \text {-butylammonium fluoride }\end{array}$


DMAP 4-(dimethylamino)pyridine

DIEA $\quad N, N$-diisopropylethylamine

DMTr-Cl $4,4^{\prime}$-dimethoxytrityl chloride

Pol polymerase

DTT dithiothreitol

EtOAc ethyl acetate

CRAB competitive replication and adduct bypass

\section{References}

1. Nordberg J, Arnér ESJ. Reactive oxygen species, antioxidants, and the mammalian thioredoxin system. Free Radical Biol. Med. 2001; 31:1287-1312. [PubMed: 11728801]

2. Finkel T. Signal transduction by reactive oxygen species. J. Cell Biol. 2011; 194:7-15. [PubMed: 21746850]

3. Stadtman ER, Berlett BS. Reactive oxygen-mediated protein oxidation in aging and disease. Drug Metab. Rev. 1998; 30:225-243. [PubMed: 9606602]

4. Droge W. Free radicals in the physiological control of cell function. Physiol. Rev. 2002; 82:47-95. [PubMed: 11773609]

5. Dizdaroglu M. Oxidatively induced DNA damage and its repair in cancer. Mutat. Res., Rev. Mutat. Res. 2015; 763:212-245. [PubMed: 25795122]

6. Dedon PC. The chemical toxicology of 2-deoxyribose oxidation in DNA. Chem. Res. Toxicol. 2008; 21:206-219. [PubMed: 18052112]

7. Amato NJ, Wang YS. Epimeric 2-deoxyribose lesions: products from the improper chemical repair of 2-deoxyribose radicals. Chem. Res. Toxicol. 2014; 27:470-479. [PubMed: 24517165]

8. Pogozelski WK, Tullius TD. Oxidative strand scission of nucleic acids: routes initiated by hydrogen abstraction from the sugar moiety. Chem. Rev. 1998; 98:1089-1107. [PubMed: 11848926]

9. Korner S, Bryant-Friedrich A, Giese B. C3'-branched thymidines as precursors for the selective generation of $\mathrm{C}^{\prime}{ }^{\prime}$-nucleoside radicals. J. Org. Chem. 1999; 64:1559-1564. [PubMed: 11674219]

10. Amato NJ, Bryant-Friedrich AC. The impact of structure on oxidatively generated DNA damage products resulting from the $\mathrm{C}^{\prime}$ '-thymidinyl radical. ChemBioChem. 2013; 14:187-190. [PubMed: 23280951]

11. Bryant-Friedrich AC. Generation of a $\mathrm{C}^{\prime}$ '-thymidinyl radical in single-stranded oligonucleotides under anaerobic conditions. Org. Lett. 2004; 6:2329-2332. [PubMed: 15228271]

12. Amato NJ, Zhai QQ, Navarro DC, Niedernhofer LJ, Wang YS. In vivo detection and replication studies of a-anomeric lesions of $2^{\prime}$-deoxyribonucleosides. Nucleic Acids Res. 2015; 43:83148324. [PubMed: 26202973]

13. Balasubramanian B, Pogozelski WK, Tullius TD. DNA strand breaking by the hydroxyl radical is governed by the accessible surface areas of the hydrogen atoms of the DNA backbone. Proc. Natl. Acad. Sci. U. S. A. 1998; 95:9738-9743. [PubMed: 9707545]

14. Roscmeycr H, Krecmerova M, Seela F. 9-(2'-Deoxy- $\beta$-D-xylofuranosyl)adenine building-blocks for solid-phase synthesis and properties of oligo( $2^{\prime}$-deoxyxylonucleotides). Helv. Chim. Acta. 1991; 74:2054-2067.

15. Rosemeyer H, Seela F. 9-(2'-Deoxy- $\beta$-D-xylofuranosyl) thymine building-blocks for solid-phase synthesis and properties of oligo(2' -deoxyxylonucleotides). Helv. Chim. Acta. 1991; 74:748-760.

16. Seela F, Worner K, Rosemeyer H. 1-(2'-Deoxy- $\beta$-D-xylofuranosyl)cytosine: base-pairing of oligonucleotides with a configurationally altered suger-phosphate backbone. Helv. Chim. Acta. 1994; 77:883-896. 
17. Seela F, Heckel M, Rosemeyer H. Xylose-DNA containing the four natural bases. Helv. Chim. Acta. 1996; 79:1451-1461.

18. Ramaswamy A, Froeyen M, Herdewijn P, Ceulemans A. Helical structure of xylose-DNA. J. Am. Chem. Soc. 2010; 132:587-595. [PubMed: 20017539]

19. Maiti M, Siegmund V, Abramov M, Lescrinier E, Rosemeyer H, Froeyen M, Ramaswamy A, Ceulemans A, Marx A, Herdewijn P. Solution structure and conformational dynamics of deoxyxylonucleic acids (dXNA): an orthogonal nucleic acid candidate. Chem. - Eur. J. 2012; 18:869-879. [PubMed: 22180030]

20. Ravindra Babu B, Raunak, Poopeiko NE, Juhl M, Bond AD, Parmar VS, Wengel J. XNA (xylo nucleic acid): a summary and new derivatives. Eur. J. Org. Chem. 2005; 2005:2297-2321.

21. Poopeiko NE, Juhl M, Vester B, Sorensen MD, Wengel J. Xylo-configured oligonucleotides (XNA, xylo nucleic acid): synthesis of conformationally restricted derivatives and hybridization towards DNA and RNA complements. Bioorg. Med. Chem. Lett. 2003; 13:2285-2290. [PubMed: 12824019]

22. Maiti M, Maiti M, Knies C, Dumbre S, Lescrinier E, Rosemeyer H, Ceulemans A, Herdewijn P. Xylonucleic acid: synthesis, structure, and orthogonal pairing properties. Nucleic Acids Res. 2015; 43:7189-7200. [PubMed: 26175047]

23. Ivanov SA, Alekseev YI, Gottikh MB. Pyrimidine oligodeoxyxylonucleotides form triplexes with purine DNA in neutral medium. Mol. Biol. 2002; 36:131-139.

24. Ivanov S, Alekseev Y, Bertrand JR, Malvy C, Gottikh MB. Formation of stable triplexes between purine RNA and pyrimidine oligodeoxyxylonucleotides. Nucleic Acids Res. 2003; 31:4256-4263. [PubMed: 12853644]

25. Petrauskene OV, Yakovleva JN, Alekseev YI, Subach FV, Babkina OV, Gromova ES. DNA duplexes containing altered sugar residues as probes of EcoRII and MvaI endonuclease interactions with sugar-phosphate backbone. J. Biomol. Struct. Dyn. 2000; 17:857-870. [PubMed: 10798530]

26. Neeley WL, Delaney S, Alekseyev YO, Jarosz DF, Delaney JC, Walker GC, Essigmann JM. DNA polymerase $\mathrm{V}$ allows bypass of toxic guanine oxidation products in vivo. J. Biol. Chem. 2007; 282:12741-12748. [PubMed: 17322566]

27. Eisenhuth R, Richert C. Convenient syntheses of $3^{\prime}$-amino- $2^{\prime}, 3^{\prime}$-dideoxynucleosides, their $5^{\prime}$ monophosphates, and $3^{\prime}$-aminoterminal oligodeoxynucleotide primers. J. Org. Chem. 2009; 74:26-37. [PubMed: 19053612]

28. Wang PC, Amato NJ, Zhai QQ, Wang YS. Cytotoxic and mutagenic properties of $O^{4}$ alkylthymidine lesions in Escherichia coli cells. Nucleic Acids Res. 2015; 43:10795-10803. [PubMed: 26400162]

29. Zhai QQ, Wang PC, Wang YS. Cytotoxic and mutagenic properties of regioisomeric $O^{2}$-, $N 3$-and $O^{4}$-ethylthymidines in bacterial cells. Carcinogenesis. 2014; 35:2002-2006. [PubMed: 24710626]

30. Delaney JC, Essigmann JM. Assays for determining lesion bypass efficiency and mutagenicity of site-specific DNA lesions in vivo. Methods Enzymol. 2006; 408:1-15. [PubMed: 16793359]

31. Hong HZ, Cao HC, Wang YS. Formation and genotoxicity of a guanine-cytosine intrastrand crosslink lesion in vivo. Nucleic Acids Res. 2007; 35:7118-7127. [PubMed: 17942427]

32. Yuan BF, Cao HC, Jiang Y, Hong HZ, Wang YS. Efficient and accurate bypass of $N^{2}-(1-$ carboxyethyl)-2' -deoxyguanosine by DinB DNA polymerase in vitro and in vivo. Proc. Natl. Acad. Sci. U. S. A. 2008; 105:8679-8684. [PubMed: 18562283]

33. Delaney JC, Essigmann JM. Context-dependent mutagenesis by DNA lesions. Chem. Biol. 1999; 6:743-753. [PubMed: 10508678]

34. Yuan BF, Jiang Y, Wang YS, Wang YS. Efficient formation of the tandem thymine glycol/8oxo-7,8-dihydroguanine lesion in isolated DNA and the mutagenic and cytotoxic properties of the tandem lesions in Escherichia coli cells. Chem. Res. Toxicol. 2010; 23:11-19. [PubMed: 20014805]

35. Barton, NH., Briggs, DEG., Eisen, JA., Goldstein, DB., Patel, NH. Evolution. Cold Spring Harbor Laboratory Press; Plainview, NY: 2007.

36. Janion C. Inducible SOS response system of DNA repair and mutagenesis in Escherichia coli. Int. J. Biol. Sci. 2008; 4:338-344. [PubMed: 18825275] 
37. Fuchs RP, Fujii S. Translesion DNA synthesis and mutagenesis in prokaryotes. Cold Spring Harbor Perspect. Biol. 2013; 5:a012682.

38. Johnson CN, Spring AM, Desai S, Cunningham RP, Germann MW. DNA sequence context conceals a-anomeric lesions. J. Mol. Biol. 2012; 416:425-437. [PubMed: 22227386] 
a. M13 genome construction

Lesion/Control

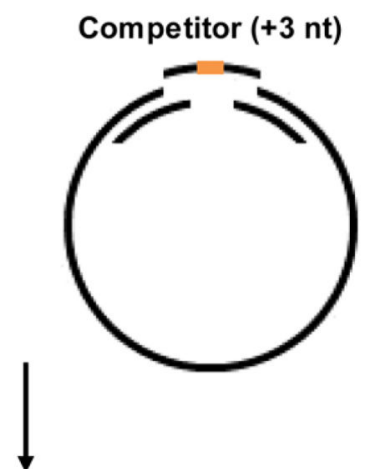

b. Electroporate genomes into $E$. coli cells
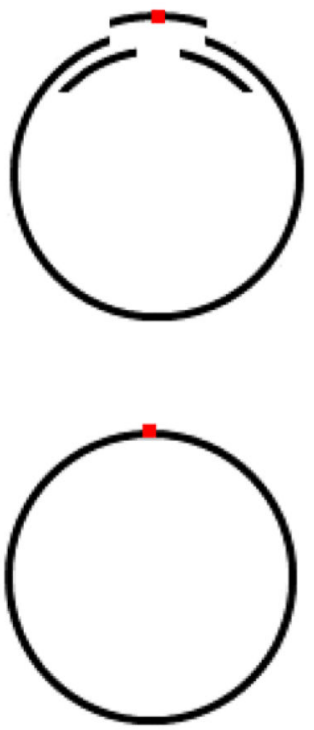

c. Progeny genome isolation

d. PCR and restriction enzyme digestion
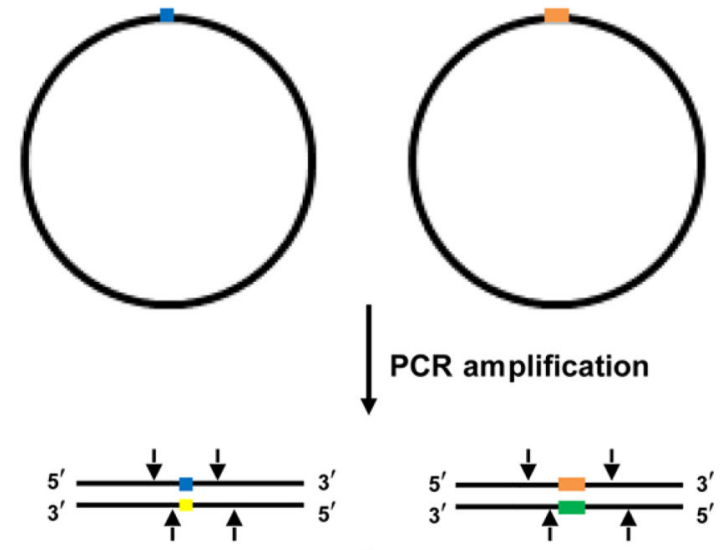

e. Replication product identification and quantification

\section{PAGE and LC-MS/MS analysis}

Figure 1.

Schematic diagram showing the experimental procedures of the competitive replication and adduct bypass (CRAB) assay. 
a (GXG)

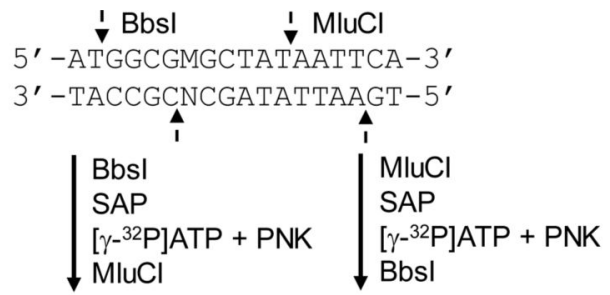

Top strand $5^{\prime}-\mathrm{AT} \mathbf{p} \mathbf{p}^{\star}$ GGCGMGCTAT AATTCAC-3' $5^{\prime}-\mathrm{AT}$ GGCGMGCTAT PAATTCA-3' Bottom strand $3^{\prime}$-TACCGCP NCGATATTAA GTG-5' 3'-TACCGC NCGATATTAAp* GT-5'

b (CXT)

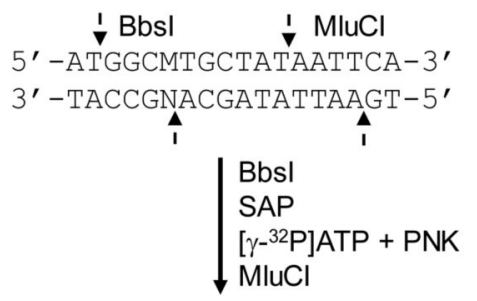

Top strand $\quad 5^{\prime}-\mathrm{AT} \mathbf{p}^{*}$ GGCMTGCTAT AATTCAC-3'

Bottom strand $3^{\prime}$-TACCGNP ACGATATTAA GTG-5'

c Top strand

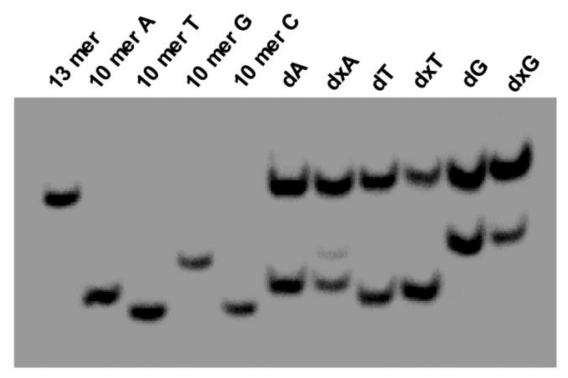

e Bottom strand d

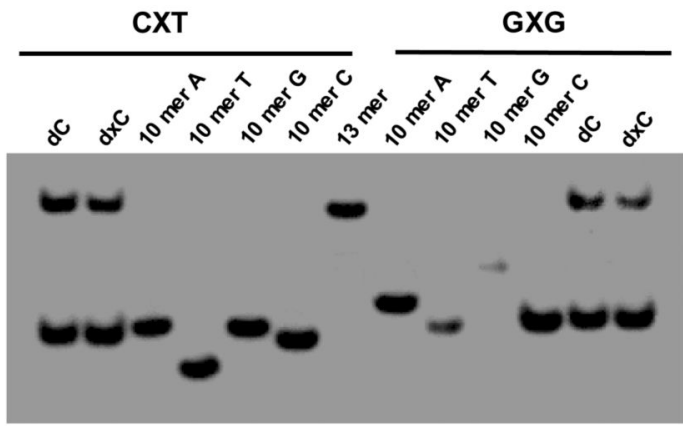

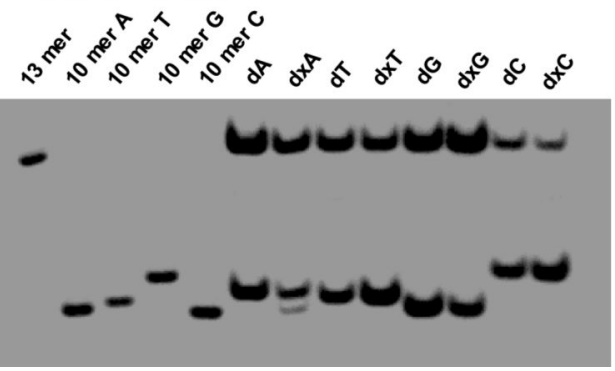

Figure 2.

Restriction digestion and radiolabeling for quantifying the bypass efficiencies and mutation frequencies of $\mathrm{C}^{\prime}$-epimeric lesions in wild-type AB1157 E. coli cells. (a and b) Schematic diagrams illustrating the selective labeling of the original lesion-bearing strand or its complementary strand via sequential restriction digestion. (c and d) Representative gel images displaying the 13-mer and 10-mer products formed from the top strand (original lesion-bearing strand) after restriction digestion of the PCR products. The 13-mer sequence is d(GGCGATAAGCTAT), which arises from the competitor genome. The 10-mer A, 10mer T, 10-mer G, and 10-mer C designate synthetic standard ODNs d(GGCGMGCTAT) and 
d(GGCMTGCTAT) for the GXG and CXT sequences, respectively, with M being A, T, G, or C. (e) Representative gel image displaying the 13-mer and 10-mer products emanating from the bottom strand (complementary to the initial lesion-containing strand) after restriction digestion of the PCR products. The 13-mer represents d(AATTATAGCTTAT) arising from the competitor genome. The 10-mer A, 10-mer T, 10-mer G, and 10-mer C designate the standard ODNs with sequences of d(AATTATAGCN) for GXG, with $\mathrm{N}$ being A, T, G, or C. 

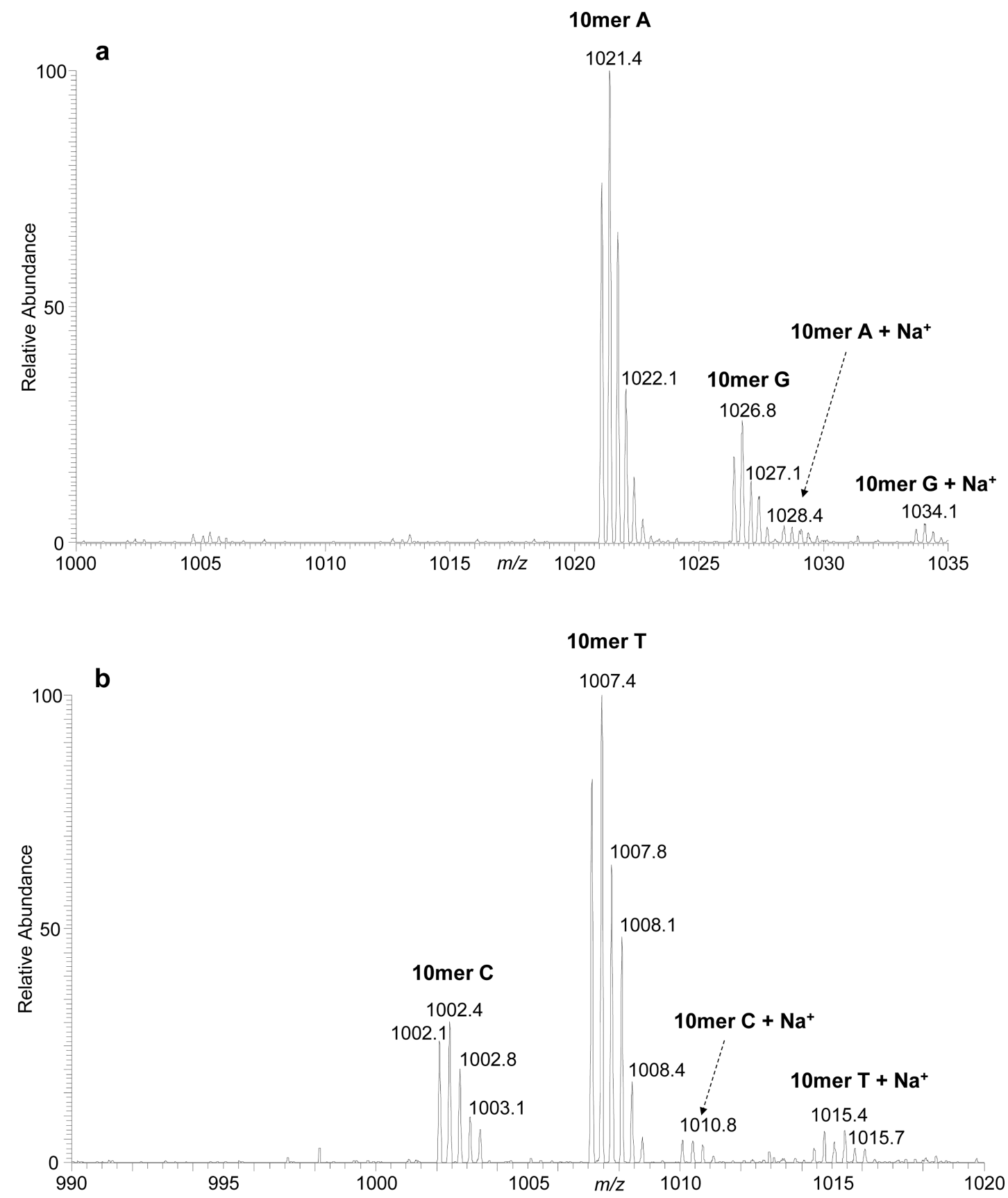

Figure 3.

Higher-resolution "ultrazoom" scan ESI-MS results for the 10-mer restriction fragments of the PCR products from the progeny genome arising from replication of the dxA-carrying single-stranded M13 genome in wild-type AB1157 cells. Shown are the $[\mathrm{M}-3 \mathrm{H}]^{3-}$ ions for the lesion-bearing strand products (a) and complementary strand products (b) from the replication of the dxA-containing plasmid. 

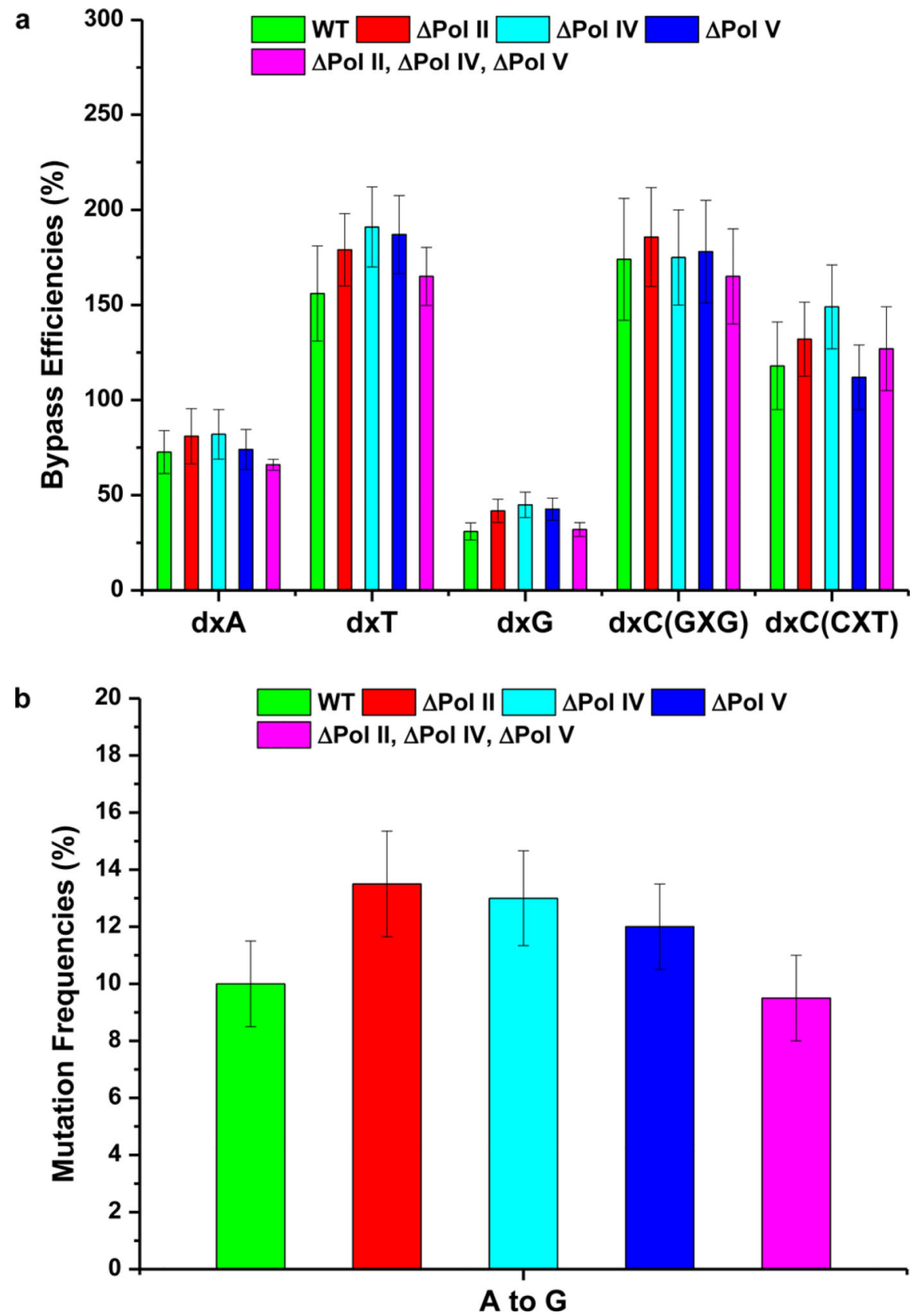

Figure 4.

Bypass efficiencies of $\mathrm{dxN}$ lesions (a) and the mutation frequencies of dxA (b) in AB1157 E. coli strains that are proficient or deficient in translesion synthesis polymerases, i.e., Pol II, Pol IV, and Pol V alone, or all three together. Because dxT, dxG, and dxC are not mutagenic, only the mutation frequencies of $\mathrm{dxA}$ are shown in panel $\mathrm{b}$. The means and standard deviations derived from three independent replication experiments are shown. 

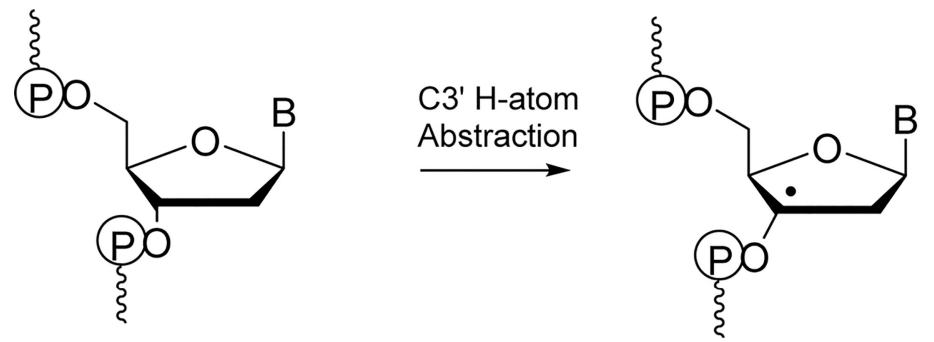

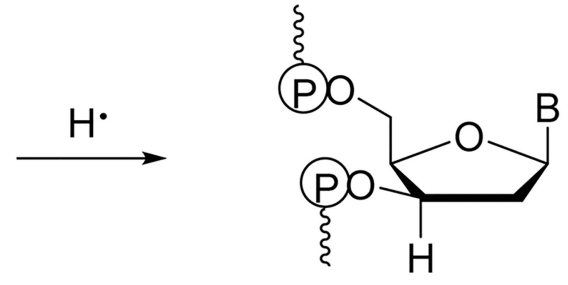

2'-deoxyxylonucleosides $(\mathrm{dxN})$

Scheme 1.

Generation of $2^{\prime}$-Deoxyxylonucleosides through the Inaccurate Chemical Repair of the C3' Radical with a Hydrogen Atom Donor ${ }^{a}$

${ }^{a} \mathrm{~B}$ indicates nucleobase. 


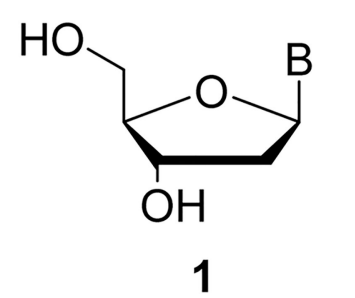

1

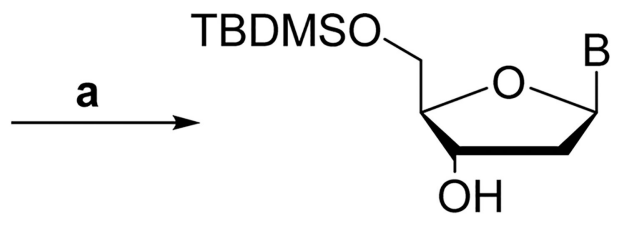<smiles>[B]C1OC2CC3CC1C3O2</smiles><smiles>CCC</smiles>

6

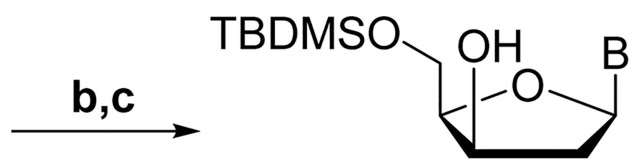

3

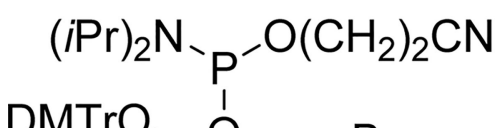

2

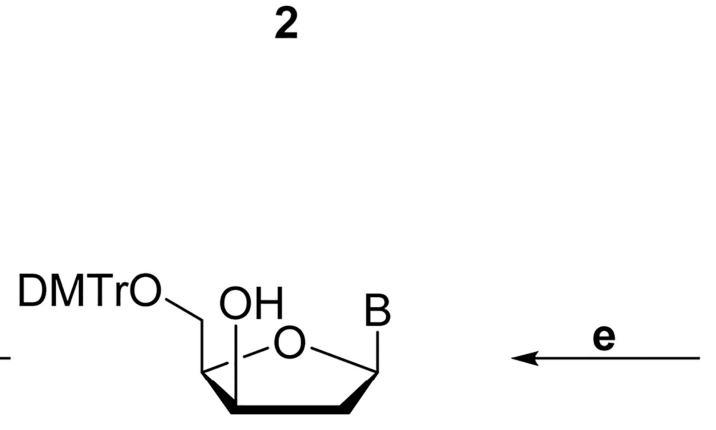

5

d

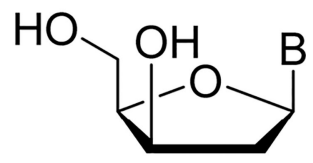

4

$$
B=(\mathbf{a}) A^{B z},(\mathbf{b}) T,(\mathbf{c}) G^{i B u}, \text { and }(\mathbf{d}) C^{B z}
$$

\section{Scheme 2.}

Synthetic Route for the Phosphoramidite Building Blocks of $2^{\prime}$-Deoxyxylonucleosides ${ }^{a}$ ${ }^{a}$ Reagents and reaction conditions: (a) TBDMS-Cl, pyridine, room temperature (rt); (b) Dess-Martin periodinane, $\mathrm{CH}_{2} \mathrm{Cl}_{2}, 0{ }^{\circ} \mathrm{C}$; (c) $\mathrm{NaBH}_{4},-60{ }^{\circ} \mathrm{C}$; (d) TBAF, THF, rt; (e) DMAP, DMTr-Cl, pyridine, rt; (f) 2-cyanoethyl- $N, N$-diisopropylchlorophosphoramidite, DIEA, $\mathrm{CH}_{2} \mathrm{Cl}_{2}$, rt. 